\title{
Approximating local observables on projected entangled pair states
}

\author{
M. Schwarz, O. Buerschaper, and J. Eisert \\ Dahlem Center for Complex Quantum Systems, Freie Universität Berlin, 14195 Berlin, Germany
}

(Received 24 August 2016; published 20 June 2017)

\begin{abstract}
Tensor network states are for good reasons believed to capture ground states of gapped local Hamiltonians arising in the condensed matter context, states which are in turn expected to satisfy an entanglement area law. However, the computational hardness of contracting projected entangled pair states in two- and higherdimensional systems is often seen as a significant obstacle when devising higher-dimensional variants of the density-matrix renormalization group method. In this work, we show that for those projected entangled pair states that are expected to provide good approximations of such ground states of local Hamiltonians, one can compute local expectation values in quasipolynomial time. We therefore provide a complexity-theoretic justification of why state-of-the-art numerical tools work so well in practice. We finally turn to the computation of local expectation values on quantum computers, providing a meaningful application for a small-scale quantum computer.
\end{abstract}

DOI: 10.1103/PhysRevA.95.060102

Introduction. Recent years have seen an explosion of interest in capturing quantum many-body systems in terms of tensor network states [1-4]. Such approaches provide powerful numerical tools for simulating strongly correlated quantum systems [2,5-8], even for fermionic systems [9-13], overcoming the notorious sign problem that mars Monte Carlo approaches. The success of such approaches is essentially rooted in the entanglement structure that ground states of gapped local Hamiltonian models exhibit: They are expected to satisfy an entanglement area law [14], originating from the locality of interactions. The insight that ground states are very atypical quantum states is often captured in the phrase that states having this entanglement pattern constitute what is called the "physical corner" of Hilbert space [15]. Indeed, the intuition that tensor network states should approximate this physical corner remarkably well is substantiated by a significant body of numerical work. In this discussion, projected entangled pair states (PEPS) [5-8,16], the higherdimensional analogs of matrix product states (MPS), take the leading role. Such PEPS not only provide numerical tools, but are also workhorses for understanding phases of matter or notions of topological order [17-19].

This development can actually be seen as a natural continuation of the established density-matrix renormalization group $(D M R G)$ method that allows one to simulate one-dimensional (1D) quantum systems essentially to machine precision $[4,20]$. For such 1D systems, a deep understanding on the functioning of tensor networks has already been reached, even to full rigor. Area laws for entanglement entropies have been proven to hold for gapped models [21], implying MPS approximations [22]. A polynomial-time classical algorithm computing an MPS approximation of ground states of gapped Hamiltonians [23] can be seen as a DMRG method with a convergence proof, at the cost of less efficiency.

But even for higher-dimensional systems, the same intuition is expected to be valid. Strictly speaking, area laws alone may not be sufficient to guarantee that PEPS are good approximations of given quantum states [24]. But the expectation that the physical corner is well approximated by PEPS, dubbed the "PEPS conjecture," is still very much in place: This expectation is usually taken for granted and constitutes the basis of an entire research field. Indeed, for higher temperatures, a variant of this conjecture is actually provably true $[25,26]$.

Having said all this, a new obstacle emerges for higherdimensional systems; one that is often seen as a key obstacle, a make-or-break issue when it comes to numerically simulating strongly correlated models with PEPS: Even though PEPS are expected to provide good approximations, they are believed to be not efficiently contractible to compute expectation values of local observables. This is backed up by a proof in worst-case complexity, stating that the contraction of two-dimensional PEPS is \#P-complete [27]. This is seen as a key burden for further developing such numerical tools. It creates a somewhat ironic situation that while the right variational principle has been identified, it may well be that one cannot compute the relevant features efficiently. This observation is also to some extent at odds with a large body of numerical evidence [5-8,11], showing that in practice, this hardness of contraction is not so much of a problem. Rather PEPS contraction gives rise to reliable results. This is even more so the case when one starts with an exact PEPS construction in the first place [28].

In this work, we contribute to clarifying this dichotomy. We show that while general PEPS may well be computationally hard to contract, this does not apply to the same extent to those PEPS that are expected to provide good approximations to ground states. Frankly put - and made more precise belowwe arrive at the following situation: Either a PEPS is a good approximation of a given ground state, and then a (quasi)polynomial time contraction is perfectly possible. Or it is not, but then the issue of contracting PEPS does not arise anyway. We will make this notion precise in the following argument.

Preliminaries. For reasons of clarity, we will first introduce some preliminaries and language that will be made use of later. A tensor of rank $r$ with dimension $D$ is a linear object in $\left(\mathbb{C}^{D}\right)^{\otimes r}$, a $D^{r}$-dimensional array of complex numbers. A tensor $T_{i_{1}, \ldots, i_{r}}$ with indices $i_{s}, 1 \leqslant s \leqslant r$, that take values in the range $1 \leqslant i_{s} \leqslant d$, may represent amplitudes of a quantum state by associating the $r$ indices to $r$ physical qudits, i.e., $|\varphi\rangle=\sum_{i_{1}, \ldots, i_{r}=1}^{d} T_{i_{1}, \ldots, i_{r}}\left|i_{1}\right\rangle \otimes \cdots \otimes\left|i_{r}\right\rangle$. Two rank $r$ tensors $S, T$ can be contracted along an index $j$ to form a rank $2 r-2$ tensor $U$ by summing over the joint index. A contracted index is called closed, whereas an uncontracted index is open. We 
consider here PEPS tensor networks based on cubic lattices of dimension $\mathrm{d}$, where vertices $v \in V$ are associated with tensors $T_{v}$. Each edge $e \in E$ between nearest neighbors connects indices of two tensors with matching bond dimension $D$, called virtual indices. Furthermore, each tensor $T_{v}$ has an open index of dimension $d$, the physical index. Consequently, a PEPS defines a quantum state vector

$$
|\psi\rangle=\sum_{i_{1}, \ldots, i_{N}=1}^{d} \mathcal{C}\left(\mathcal{T}_{i_{1}, \ldots, i_{N}}\right)\left|i_{1}\right\rangle \otimes \cdots \otimes\left|i_{N}\right\rangle
$$

where the contraction is over all closed indices for each $i_{1}, \ldots, i_{N}$, according to the contraction value $\mathcal{C}(\mathcal{T})=$ $\sum_{i_{e}=1, e \in E}^{D} \prod_{v \in V} T_{v ; i_{e}}$. The term "projected entangled pair state" (PEPS) derives from the following alternative view of a tensor network: Put a maximally entangled pair of qudits along each edge $e \in E$, i.e. $\left|\phi_{e}\right\rangle=D^{-1 / 2} \sum_{i=1}^{D}|i, i\rangle$. Then the tensors $T_{v}$ can be viewed as linear maps from the $r$ inbound virtual indices of dimension $D$ at each vertex $v$ to the single physical index of dimension $d$ called the PEPS "projector"

$$
A_{v}=\sum_{i=1}^{d} \sum_{j_{1}, \ldots, j_{r}=1}^{D} T_{v ; i, j_{1}, \ldots, j_{r}}|i\rangle\left\langle j_{1}, \ldots, j_{r}\right| .
$$

Using this map, the state vector of the PEPS can be written as

$$
|\psi\rangle=\bigotimes_{v \in V} A_{v} \bigotimes_{e \in E}\left|\phi_{e}\right\rangle
$$

A PEPS is called injective [16,29] if and only if each PEPS projector $A_{v}$ has a left inverse, i.e., $A_{v}^{-1} A_{v}=\mathrm{id}$, where $A_{v}^{-1}$ is the Moore-Penrose inverse [30]. Intuitively, this means that we can achieve any action on the virtual indices by acting on the physical systems. Any noninjective PEPS is $\epsilon$-close to an injective one for any $\epsilon>0$. This implies that "almost all" PEPS are injective. The main result about injective PEPS is a standard construction of a frustration-free local parent Hamiltonian, which has this PEPS as its unique ground state [29]. We say a PEPS defined by local tensors $\left\{A_{v}\right\}_{0 \leqslant v \leqslant N}$ has a uniformly gapped parent Hamiltonian $H_{*}$ with spectral gap $\Delta_{*}$, if every member $H_{t}$ of the family $\left\{H_{t}\right\}_{0 \leqslant t \leqslant N}$ of the parent Hamiltonian of the sub-PEPS $\left\{A_{v}\right\}_{0 \leqslant v \leqslant t}$ has a spectral gap $\Delta_{t} \geqslant \Delta_{*}[31-33]$.

PEPS conjecture. Tensor networks and, in particular, projected entangled pair states are generally expected to describe ground states of gapped many-body models exceedingly well. Recent years of numerical and analytical work on tensor network states have largely clarified that the "physical corner" of Hilbert space may indeed be well parametrized by PEPS. Still, interestingly, a "PEPS conjecture" that specifies in what precise way one expects PEPS to provide good approximations has not yet been formulated in written form, albeit being common knowledge in the community. Here we present two readings of what could reasonably be called a PEPS conjecture. We consider local Hamiltonians $H=\sum_{i} h_{i}$ defined on a d-dimensional regular lattice of spins with a constant spectral gap $\Delta>0$ above the unique ground state $\rho$. Furthermore, we are interested in arbitrary observables $O_{X}$ as long as the number of sites in their support $X$ is upper bounded by a constant $k$. In particular, observables supported on multiple disconnected regions are perfectly covered, e.g., two-point correlation functions. The first conjecture merely states that PEPS are good approximations for ground states of gapped models.

Conjecture 1 (Weak PEPS conjecture). There exists a PEPS $\omega$ with bond dimension $D=O\left(\operatorname{poly}\left(N, \epsilon^{-1}\right)\right)$ such that for all $O_{X}$ and any $\epsilon>0,\left|\operatorname{tr}\left(O_{X} \rho\right)-\operatorname{tr}\left(O_{X} \omega\right)\right|<\epsilon$.

This is provably true for 1D systems [21-23], even in the stronger incarnation that such a MPS approximation exists satisfying $\|\omega-\rho\|_{1}<\epsilon$, implying the above. Similarly, one can ask the PEPS $\omega$ to approximate the ground state $\rho$ in relative entropy $S(\omega \| \rho) \leqslant \epsilon^{2} / 2$, which implies the above by virtue of Pinsker's inequality $\|\omega-\rho\|_{1} \leqslant[2 S(\omega \| \rho)]^{1 / 2}$. For systems with $d>1$, the closest result to Conjecture 1 we are aware of is the one presented in Ref. [25], which uses a specific assumption on the density of states to find $D=e^{O\left[\log _{2}(N / \epsilon)^{\mathrm{d}+1}\right]}$, which is quasipolynomial in $N / \epsilon$ for constant $\mathrm{d}$.

While this may be reasonable, the conjecture misses the point that it does not necessarily capture key properties of the true ground state. Again for 1D systems, injectivity of the MPS will readily imply exponentially decaying correlations. This, however, is in general no longer the case for PEPS [34] and hence a new subtlety arises. But we know from Hastings [35,36] that ground states of gapped Hamiltonians in all lattice dimensions do have exponentially decaying correlations. Conjecture 1 includes situations of the kind where a state of the form $\lambda \rho+(1-\lambda) \eta$ for $\lambda \geqslant 1-\epsilon / 2$ would provide an approximation for the ground state $\rho$, even if the latter state $\eta$ has correlation properties very different from those of $\rho$, possibly not even decaying with the distance. It is hence most natural to require the PEPS approximating a ground state of a gapped model to have itself a uniformly gapped parent Hamiltonian, from which one can in turn infer about exponential decay of correlations. One can hence argue that reasonable approximations of ground states of gapped models should themselves be ground states of gapped models, which is a very natural, but technically slightly stronger conjecture.

Conjecture 2 (Strong PEPS conjecture). There exists an injective PEPS $\omega$ with bond dimension $D=O\left(\operatorname{poly}\left(N, \epsilon^{-1}\right)\right)$ with a parent Hamiltonian $H_{*}$ with uniform constant spectral gap $\Delta_{*}>0$, such that for all $O_{X}$ and any constant $\epsilon>0$, $\left|\operatorname{tr}\left(O_{X} \rho\right)-\operatorname{tr}\left(O_{X} \omega\right)\right|<\epsilon$.

Importantly, this conjecture is again provably true even in higher dimensions for all states that are in the same phase as a product state-so which are the trivial phase, viewed from a perspective of topological order: The relevant states can then be quasiadiabatically prepared [37] from products, giving rise to a short-ranged quantum circuit. Following previous work [31-33], we restrict ourselves to the subset of models exhibiting a uniform spectral gap, i.e., we ask for a constant spectral gap lower bound for a family of parent Hamiltonians related to the parent Hamiltonian of the input PEPS. This is a natural and common feature for gapped local models, and can be proven to hold for classes of PEPS. At the same time, this property does not directly follow from the PEPS conjectures as both examples as well as counterexamples are known.

Main result. We now turn to showing how expectation values of local observables can be (quasi-)efficiently approximated on PEPS which are the ground states of local Hamiltonians. We will assume constituents referred to as 
"spins" to have finite dimension $d$. We discuss contraction properties of PEPS in general terms, which can of course be seen as PEPS that approximate ground states via Conjecture 2.

Theorem 1 (Computation of observables). Let $\omega$ be an unnormalized, injective PEPS defined on a (constant) ddimensional lattice of $N$ spins with bond dimension $D$, and physical dimension $d$, such that its parent Hamiltonian $H_{*}$ is uniformly gapped with constant spectral gap $\Delta_{*}>0$. Let $\left\{A_{i}\right\}$ be the collection of local tensors specifying the PEPS, and let $\kappa_{*}=\max _{i} \kappa\left(A_{i}\right)$ be an upper bound on the condition number. Let $O_{X}$ be an observable supported on $|X|<k$ sites for constant $k$. Then an approximation $\widetilde{O_{X}}$ of the expectation value $\left\langle\omega\left|O_{X}\right| \omega\right\rangle /\langle\omega \mid \omega\rangle$ such that

$$
\left|\left\langle\omega\left|O_{X}\right| \omega\right\rangle /\langle\omega \mid \omega\rangle-\widetilde{O_{X}}\right| \leqslant \varepsilon,
$$

can be computed in time $(D d)^{O\left(\ell^{\mathrm{d}}\right)}$ on a deterministic classical computer, and time $\tilde{O}\left(\ell^{\mathrm{d}} / \varepsilon^{2}\right)$ and $O(\operatorname{polylog}(\ell / \varepsilon))$ depth on a quantum computer, where

$$
\ell \in O\left(\frac{2 \ln \left(\kappa_{*}\right)+\ln \left(\varepsilon^{-1}\right)+\ln \left(\left\|O_{X}\right\|\right)}{\Delta_{*}}\right) .
$$

That is, the computation is possible in quasipolynomial deterministic time or polylogarithmic quantum depth for polynomially scaling $\varepsilon^{-1}$ and $\kappa_{*}$, and constant $\mathrm{d}, \Delta_{*}>0$. Or in polylogarithmic quantum time for polynomial $\kappa_{*}$ and polylogarithmic $\varepsilon^{-1}$ and constant $\mathrm{d}, \Delta_{*}>0$. Or in constant deterministic time for constant $\mathrm{d}, \Delta_{*}, \kappa_{*}, \varepsilon>0$. In the special case of $\mathrm{d}=1$, the runtime of the algorithm scales polynomially in the system size, as expected for MPS. Note that the set $X$ does not have to be simply connected, so that our theorem covers observables $O_{X}$ reflecting correlation functions. A related result has also been shown in Ref. [38] assuming local topological quantum order (LTQO), a condition implying exponential decay of correlations. Our result only uses the exponential clustering theorem-which is provably true in all finite dimensions $[35,36]$ - while LTQO is not known to hold in this generality.

Proof sketch of Theorem 1. We present here the core argument of the proof, with further details and the explicit algorithms stated in Supplemental Material [39]. The key idea is to disentangle a boundary of size $O\left(\ell^{\mathrm{d}-1}\right)$ of PEPS tensors $\left\{A_{i}\right\}$ around the observable $O_{X}$ from the PEPS step by step Fig. 1, while bounding the additive error in estimating the expectation value of $O_{X}$ introduced in each step. In this way, we achieve an overall error bound that scales linearly with the number of tensors removed, that is $O\left(\ell^{\mathrm{d}-1}\right)$ times the per-step error. A key tool we use is the exponential clustering theorem, which is known to hold in all finite dimensions $[35,36]$. To some extent, our approach can also be viewed as partially inverting the PEPS preparation algorithms as discussed in Refs. [31-33]. For an unnormalized, injective PEPS $|\omega\rangle$, specified by the collection of tensors $\left\{A_{i}\right\}$, with a gapped, local parent Hamiltonian $H_{*}$ [29], we define a sequence of parent Hamiltonians $H_{i}, 0 \leqslant i \leqslant N$, on the same d-dimensional lattice, with formally normalized ground states

$$
\left|\omega_{i}\right\rangle=\frac{A_{i} \cdots A_{1}|\phi\rangle^{\otimes n}}{\| A_{i} \cdots A_{1}|\phi\rangle^{\otimes n} \|},
$$

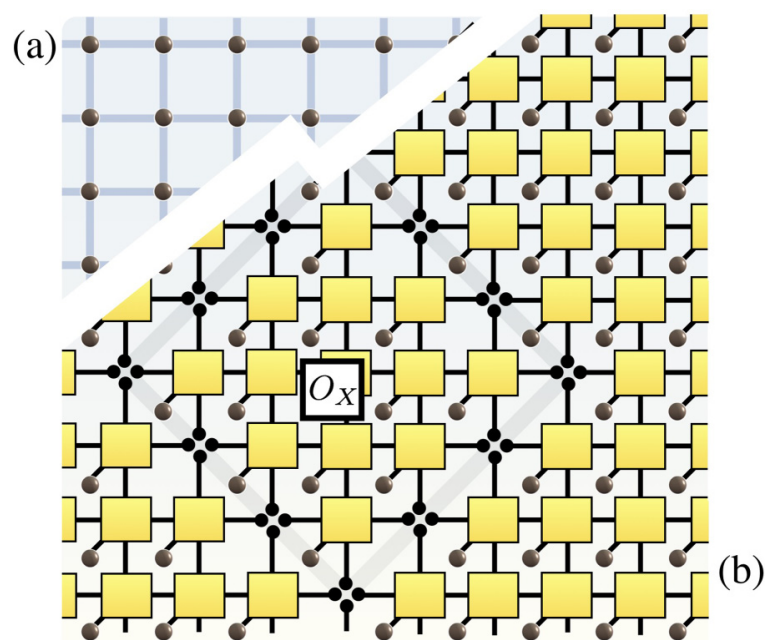

FIG. 1. (a) A two-dimensional quantum lattice model. If the model is gapped, its ground state is conjectured to be wellapproximated by a PEPS. (b) PEPS tensors mapping virtual to physical indices.

so that $H_{N}=H_{*}, n=O(N)$ denoting the number of edges. We assume that the last $N_{b}=O\left(\ell^{\mathrm{d}-1}\right)$ tensors in the sequence $\left(A_{i}\right)_{0 \leqslant i \leqslant N}$ constitute a boundary at graph theoretical distance $\ell$ on the lattice around observable $O_{X}$ (this sequence can always be constructed for an injective PEPS [29,34]). By assumption $H_{*}$ is uniformly gapped by $\Delta_{*}$, therefore $\Delta\left(H_{i}\right) \geqslant \Delta_{*}$ for all $H_{i}$, following Refs. [31-33]. The exponential clustering theorem [35,36] applies to each $H_{i}$, such that for each $i$ and fixed $O_{X}$

$$
\begin{aligned}
& \left|\left\langle\omega_{i}\left|O_{X} \otimes O_{i}\right| \omega_{i}\right\rangle-\left\langle\omega_{i}\left|O_{X}\right| \omega_{i}\right\rangle\left\langle\omega_{i}\left|O_{i}\right| \omega_{i}\right\rangle\right| \\
& \leqslant e^{-O\left(\ell \Delta_{*}\right)}\left\|O_{X}\right\|\left\|O_{i}\right\| .
\end{aligned}
$$

Choosing $O_{i}=\left(A_{i}^{-1}\right)^{\dagger} A_{i}^{-1}$ to disentangle the PEPS tensor $A_{i}$, and dividing by $\| A_{i}^{-1}\left|\omega_{i}\right\rangle \|^{2}=\left\langle\omega_{i}\left|\left(A_{i}^{-1}\right)^{\dagger} A_{i}^{-1}\right| \omega_{i}\right\rangle$ gives

$$
\begin{aligned}
& \left|\frac{\left\langle\omega_{i}\left|O_{X} \otimes\left(A_{i}^{-1}\right)^{\dagger} A_{i}^{-1}\right| \omega_{i}\right\rangle}{\left\langle\omega_{i}\left|\left(A_{i}^{-1}\right)^{\dagger} A_{i}^{-1}\right| \omega_{i}\right\rangle}-\left\langle\omega_{i}\left|O_{X}\right| \omega_{i}\right\rangle\right| \\
& \leqslant e^{-O\left(\ell \Delta_{*}\right)}\left\|O_{X}\right\| \frac{\left\|\left(A_{i}^{-1}\right)^{\dagger} A_{i}^{-1}\right\|}{\left\langle\omega_{i}\left|\left(A_{i}^{-1}\right)^{\dagger} A_{i}^{-1}\right| \omega_{i}\right\rangle},
\end{aligned}
$$

which can be upper bounded by $e^{-O\left(\ell \Delta_{*}\right)}\left\|O_{X}\right\| \kappa\left(A_{i}\right)^{2}$, using $\kappa(A)=\kappa\left(A^{-1}\right)$. Equipped with this bound, we can iteratively approximate the expectation value $\left\langle\omega\left|O_{X}\right| \omega\right\rangle /\langle\omega \mid \omega\rangle$ using the triangle inequality $O\left(\ell^{\mathrm{d}-1}\right)$ times and bounding the error as we move from $\left|\omega_{N}\right\rangle=|\omega\rangle / \||\omega\rangle \|$ to $\left|\omega_{N-N_{b}}\right\rangle=:\left|\omega_{*}\right\rangle$ :

$$
\left|\left\langle\omega_{*}\left|O_{X}\right| \omega_{*}\right\rangle-\frac{\left\langle\omega\left|O_{X}\right| \omega\right\rangle}{\langle\omega \mid \omega\rangle}\right| \leqslant \ell^{\mathrm{d}-1} e^{-O\left(\ell \Delta_{*}\right)} \kappa_{*}^{2}\left\|O_{X}\right\| .
$$

For any given error $\varepsilon>0$ we can choose a sufficiently large $\ell$ as in Eq. (2), defining a patch of the tensor network of radius $\ell$ around the observable $O_{X}$ that can be disentangled from the rest of the state while not changing the expectation value of $O_{X}$ by more than $\varepsilon$. For constant lattice dimension d, well-conditioned $\kappa_{*}=O(\operatorname{poly}(N)), \varepsilon=1 / O(\operatorname{poly}(N))$, and $\left\|O_{X}\right\|=O(\operatorname{poly}(N))$, a choice of $\ell=O(\log (N))$ suffices. We 
will now turn to showing how to compute this expectation value in quasipolynomial time $O\left(2^{(\log N)^{O(1)}}\right)$. We write the state vector $\left|\omega_{*}\right\rangle$ as the formally normalized PEPS $\left|\omega_{*}\right\rangle=$ $A_{N-N_{b}} \cdots \cdot A_{1}|\phi\rangle^{\otimes n} / \| A_{N-N_{b}} \cdots A_{1}|\phi\rangle^{\otimes n} \|$. Since we have disentangled all PEPS tensors $A_{i}$ on a boundary surface, the PEPS $\left|\omega_{*}\right\rangle$ is a tensor product of the patch $\left|\omega_{P}\right\rangle$ and the remainder PEPS $\left|\omega_{R}\right\rangle,\left|\omega_{*}\right\rangle=\left|\omega_{R}\right\rangle \otimes\left|\omega_{P}\right\rangle$,

$$
\left|\omega_{*}\right\rangle=\frac{\bigotimes_{r \in R} A_{r}|\phi\rangle^{\otimes n_{R}}}{\| \bigotimes_{r \in R} A_{r}|\phi\rangle^{\otimes n_{R}} \|} \otimes \frac{\bigotimes_{p \in P} A_{p}|\phi\rangle^{\otimes n_{P}}}{\| \bigotimes_{p \in P} A_{p}|\phi\rangle^{\otimes n_{P}} \|} .
$$

Since $O_{X}$ acts only on $\left|\omega_{P}\right\rangle$ and $\left\langle\omega_{R} \mid \omega_{R}\right\rangle=1$, one gets

$$
\left\langle\omega_{*}\left|O_{X}\right| \omega_{*}\right\rangle=\left\langle\omega_{P}\left|O_{X}\right| \omega_{P}\right\rangle=\frac{\left\langle\phi\left|\bigotimes_{p \in P} A_{p}^{\dagger} O_{X} A_{p}\right| \phi\right\rangle}{\left\langle\phi\left|\bigotimes_{p \in P} A_{p}^{\dagger} A_{p}\right| \phi\right\rangle} .
$$

The left tensor factor $\left|\omega_{R}\right\rangle$ in Eq. (6) has been reduced to a scalar 1 by construction in this step. We have hence removed the need to contract any part of the PEPS outside of the patch or to compute a global norm. All remaining computations can be performed locally. The tensor networks in both the numerator as well as the denominator in Eq. (7) can be contracted exactly in time $(D d)^{O\left(\ell^{\mathrm{d}}\right)}$ by summing over all $2\left(\mathrm{~d} \ell^{\mathrm{d}}\right)$ indices of dimension $D$ and $\ell^{\mathrm{d}}$ indices of physical dimension $d$, resulting in $(D d)^{O\left(\ell^{\mathrm{d}}\right)}$ terms, for constant $\mathrm{d} \geqslant 2$ and constant $\ell$ in polynomial and for $\ell=O(\log (N))$ in quasipolynomial time.

Computation of expectation values on a quantum computer. We have seen that the expectation value of a local observable can be approximated by computing the expectation value on a small patch of radius $\ell=O(\log (N))$ instead of the full PEPS. This fact suggests that the desired expectation value could also be computed on a quantum computer acting on only $O(\log (N))$ qubits, instead of simulating the full system of size $N$. Indeed, this observation contributes to the discussion on feasible applications of a small quantum computer consisting of tens or hundreds, but not thousands or more qubits.

Hardness of tensor network contraction and implications for practical algorithms. The result obtained here is not in conflict with the known hardness result [27] for contracting general PEPS, an argument that we lay out in more detail in Supplemental Material. The key reason is the restriction to PEPS which are ground states of local Hamiltonians with constant spectral gap. This assumption significantly reduces the computional power of a PEPS oracle viewed as a computational resource [27]. It is key to the above argument that it readily gives rise to a constructive quasipolynomial contraction algorithm. For clarity, this algorithm is again fleshed out in great detail in Supplemental Material. The results established here contribute to providing evidence of why existing numerical contraction methods of PEPS perform so well in numerical studies.

Injective PEPS with uniformly gapped parent Hamiltonians satisfy a variant of local topological quantum order. In Ref. [38] it is shown that parent Hamiltonians of translationally invariant, injective MPS satisfy the LTQO condition. Combining Eqs. (6) and (5) yields

$$
\left|\frac{\left\langle\omega_{P}\left|O_{X}\right| \omega_{P}\right\rangle}{\left\langle\omega_{P} \mid \omega_{P}\right\rangle}-\frac{\left\langle\omega\left|O_{X}\right| \omega\right\rangle}{\langle\omega \mid \omega\rangle}\right| \leqslant\left\|O_{X}\right\| \ell^{\mathrm{d}-1} e^{-O\left(\ell \Delta_{*}\right)} \kappa_{*}^{2},
$$

which might superficially appear to satisfy the LTQO condition in Ref. [38, Def. 2], but actually does not. Rather, the two statements differ in the type of boundary terms allowed: while the cited LTQO condition only allows one to strictly remove local Hamiltonian terms along a boundary surface, the parent Hamiltonians constructed in our proof actually do add boundary terms to enforce the uniqueness of the ground state. Thus, in this sense our proof does not imply LTQO for injective PEPS as defined, but rather a variant of LTQO with unique ground states, which may be of independent interest.

Conclusion and outlook. In this work, we have shown that expectation values of local observables in PEPS that naturally approximate ground states of Hamiltonian models can be computed in quasipolynomial time. In this way, we contribute a complexity-theoretic picture to the widely observed common observation that in numerical approaches, such computations are well feasible. It remains a very interesting problem for future work to extend our results to $G$-injective ("groupinjective") PEPS. We hence contribute to demystifying the complexity of contracting tensor network states, coming to the conclusion that the situation for higher-dimensional systems is not that different compared to 1D systems, for which the DMRG approach provides the workhorse of numerical studies. Our work can be seen as a further invitation to the program of capturing condensed matter systems in terms of tensor network states.

Acknowledgments. We thank I. Arad, M. Kastoryano, Y. Ge, Z. Landau, A. Molnár, N. Schuch, and F. Verstraete for discussions and the ERC (TAQ), the EC (SIQS, RAQUEL, AQuS), the Templeton Foundation, the DFG (EI 519/7-1, CRC 183), and the Alexander von Humboldt Foundation for support.
[1] R. Orus, Ann. Phys. (NY) 349, 117 (2014).

[2] F. Verstraete, J. I. Cirac, and V. Murg, Adv. Phys. 57, 143 (2008).

[3] J. Eisert, in Emergent Phenomena in Correlated Matter, edited by E. Pavarini, E. Koch, and U. Schollwöck, Modeling and Simulation (Forschungszentrum Jülich Zentralbibliothek, Verlag, Jülich, 2013), Vol. 3, p. 520.

[4] U. Schollwöck, Ann. Phys. (NY) 326, 96 (2011).

[5] T. Picot, M. Ziegler, R. Orus, and D. Poilblanc, Phys. Rev. B 93, 060407 (2016).
[6] J. Jordan, R. Orus, G. Vidal, F. Verstraete, and J. I. Cirac, Phys. Rev. Lett. 101, 250602 (2008).

[7] H. N. Phien, J. A. Bengua, H. D. Tuan, P. Corboz, and R. Orus, Phys. Rev. B 92, 035142 (2015).

[8] M. Lubasch, J. I. Cirac, and M.-C. Banuls, Phys. Rev. B 90, 064425 (2014).

[9] P. Corboz, G. Evenbly, F. Verstraete, and G. Vidal, Phys. Rev. A 81, 010303(R) (2010).

[10] P. Corboz and G. Vidal, Phys. Rev. B 80, 165129 (2009). 
[11] P. Corboz, Phys. Rev. B 93, 045116 (2016).

[12] C. Pineda, T. Barthel, and J. Eisert, Phys. Rev. A 81, 050303 (2010).

[13] P. Corboz, R. Orus, B. Bauer, and G. Vidal, Phys. Rev. B 81, 165104 (2010).

[14] J. Eisert, M. Cramer, and M. B. Plenio, Rev. Mod. Phys. 82, 277 (2010).

[15] D. Poulin, A. Qarry, R. Somma, and F. Verstraete, Phys. Rev. Lett. 106, 170501 (2011).

[16] F. Verstraete and J. I. Cirac, arXiv:cond-mat/0407066.

[17] N. Schuch, D. Perez-Garcia, and I. Cirac, Phys. Rev. B 84, 165139 (2011).

[18] O. Buerschaper, Ann. Phys. (NY) 351, 447 (2014).

[19] N. Schuch, J. I. Cirac, and D. Perez-Garcia, Ann. Phys. (NY) 325, 2153 (2010).

[20] S. R. White, Phys. Rev. Lett. 69, 2863 (1992).

[21] M. B. Hastings, J. Stat. Mech. (2007) P08024.

[22] N. Schuch, M. M. Wolf, F. Verstraete, and J. I. Cirac, Phys. Rev. Lett. 100, 030504 (2008).

[23] Z. Landau, U. Vazirani, and T. Vidick, Nat. Phys. 11, 566 (2015).

[24] Y. Ge and J. Eisert, New J. Phys. 18, 083026 (2016).

[25] A. Molnár, N. Schuch, F. Verstraete, and J. I. Cirac, Phys. Rev. B 91, 045138 (2015).

[26] M. Kliesch, C. Gogolin, M. J. Kastoryano, A. Riera, and J. Eisert, Phys. Rev. X 4, 031019 (2014).

[27] N. Schuch, M. M. Wolf, F. Verstraete, and J. I. Cirac, Phys. Rev. Lett. 98, 140506 (2007).

[28] D. Poilblanc, N. Schuch, D. Perez-Garcia, and J. I. Cirac, Phys. Rev. B 86, 014404 (2012).
[29] D. Perez-Garcia, F. Verstraete, J. I. Cirac, and M. M. Wolf, Quant. Inf. Comp. 8, 650 (2008).

[30] The injectivity condition also holds if the left inverse exists only after blocking of a constant number of tensors, merging a constant number of adjacent physical indices in turn. On a d-dimensional lattice, it is also clear from dimension counting alone that, after blocking, the PEPS projector maps the virtual space of smaller dimension to the physical space of larger dimension.

[31] M. Schwarz, K. Temme, and F. Verstraete, Phys. Rev. Lett. 108, 110502 (2012).

[32] M. Schwarz, K. Temme, F. Verstraete, D. Perez-Garcia, and T. S. Cubitt, Phys. Rev. A 88, 032321 (2013).

[33] Y. Ge, A. Molnar, and J. I. Cirac, Phys. Rev. Lett. 116, 080503 (2016).

[34] F. Verstraete, M. M. Wolf, D. Perez-Garcia, and J. I. Cirac, Phys. Rev. Lett. 96, 220601 (2006).

[35] M. B. Hastings, Phys. Rev. B 69, 104431 (2004).

[36] B. Nachtergaele and R. Sims, Commun. Math. Phys. 265, 119 (2006).

[37] S. Bachmann, S. Michalakis, B. Nachtergaele, and R. Sims, Commun. Math. Phys. 309, 835 (2012).

[38] J. I. Cirac, S. Michalakis, D. Pérez-García, and N. Schuch, Phys. Rev. B 88, 115108 (2013).

[39] See Supplemental Material at http://link.aps.org/supplemental/ 10.1103/PhysRevA.95.060102 for giving full proof of Theorem 1 , explicitly stating the classical contraction algorithm implied, and providing a more detailed discussion on the hardness of tensor network contraction and the computation of expectation values on a quantum computer. 